Orinoquia, Julio-Diciembre 2019;23(2):63-70

ISSN electrónico 2011-2629.

ISSN impreso 0121-3709.

https://doi.org/10.22579/20112629.570

\title{
Reproducción en cautiverio de vizcaína Curimata mivartii con extracto pituitario de carpa
}

\author{
Captivity reproduction of vizcaina Curimata mivartii induced with \\ extract of carp pituitary
}

\section{Reprodução em cativeiro de vizcaína Curimata mivartii com extrato de hipófise de carpa}

César Montes-Petro ${ }^{1}$; Víctor Atencio-García ${ }^{2}$; Ana Estrada-Posada ${ }^{3}$; Jonny Yepes-Blandón ${ }^{4}$

1 Prof. Acuiclt, MSc, Grupo de Investigación en Peces Nativos-GIPEN, Piscícola San Silvestre S.A., Barrancabermeja Santander

2 Ing. Pesquera, Esp, MSc, Universidad de Córdoba, Facultad de Medicina Veterinaria y Zootecnia, Departamento de Ciencias Acuícolas, Instituto de Investigaciones Piscícolas

3 Biol, MSc, (c)PhD, ISAGEN S.A

4 Zoo, MSc, (c)PhD, Grupo de Investigación en Peces Nativos-GIPEN, Piscícola San Silvestre S.A., Barrancabermeja Santander

Email: investigacionpisansilvestre@gmail.com

\begin{abstract}
Resumen
Con el objetivo de reproducir vizcaína Curimata mivartii, ejemplares de dos años de edad ( $\mathrm{n}=54)$, mantenidos en estanques en tierra $\left(0,1 \mathrm{pez} / \mathrm{m}^{2}\right)$ fueron inducidos con extracto pituitario de carpa (EPC) (Argent, USA) a razón de $6 \mathrm{mg} / \mathrm{Kg}$ de peso para las hembras en dos aplicaciones (10\% y 90\% con 12 horas entre aplicación); mientras que los machos fueron inducidos con 4,8 mg/Kg EPC en dosis única. Antes de la inducción hormonal a cada hembra le fue realizada biopsia para verificar su estado de maduración gonadal. La muestra de ovocitos fue tratada con solución Serra (Chemí, Colombia) y después de tres minutos, con la ayuda de un estereoscopio de luz, (Leica, EZ4 W, Alemania) en objetivo de 4x, se determinó posición de la vesícula germinal (céntrico, migrando, periférico, sin núcleo). El análisis de la biopsia ovárica mostró que las hembras se encontraban en maduración final. Los machos inducidos se encontraron en fase de espermiación; los cuales a ligera presión abdominal liberaron líquido seminal. Se estimó el índice de ovulación, fecundidad (absoluta y relativa), diámetro de los ovocitos y volumen seminal. El porcentaje de fertilidad fue estimado a las cuatro horas post-eclosión (hpe) y el porcentaje de eclosión fue estimado a las 10 hpe. Las hembras ovularon entre 5 y 6 horas $\left(28 \pm 1,0^{\circ} \mathrm{C}\right)$ e inmediatamente los huevos fueron obtenidos por extrusión, así como el semen. La fecundidad absoluta fue de 178331,6 228773,7 ovocitos/

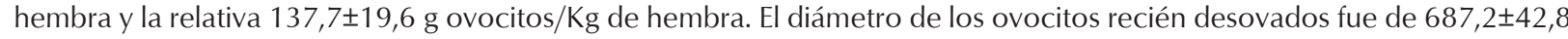
um. La fertilidad fue de 90,7 $\pm 1,4 \%$ y la eclosión de $85,3 \pm 1,5 \%$. Los resultados permiten concluir que EPC a dosificación de


$6 \mathrm{mg} / \mathrm{Kg}$ de peso es un buen inductor de la reproducción de vizcaína, con alto índice de ovulación y altos porcentajes de fertilidad y eclosión.

Palabras claves: Curimatidae; desove; eclosión; fecundidad

\begin{abstract}
With the aim of reproducing the vizcaina Curimata mivartii, two-year-old specimens $(n=54)$, kept in ponds on land $(0,1$ fish $/ \mathrm{m}^{2}$ ) were induced with carp pituitary extract (EPC) (Argent, USA) at a doses of $6 \mathrm{mg} / \mathrm{Kg}$ of weight for females in two applications (10\% and 90\% with 12 hours between applications), while males were induced with 4,8 mg / Kg EPC in a single dose. Before the hormonal induction, each female underwent an ovarian biopsy to verify the oocytes maturation stage. The oocyte sample was treated with Serra solution (Chemi, Colombia) and after three minutes, with the help of a light stereoscope (Leica, EZ4 W, Germany) in 4x objective, the position of the germinal vesicle was determined (central, migrating, peripheral, breakdown). The analysis of the ovarian biopsy showed that the females were in final maturation. Induced males were found in the spermiation phase; which at light abdominal pressure released seminal fluid. The ovulation index, fecundity (absolute and relative), oocyte diameter and seminal volume were estimated. The percentage of fertility was estimated at four hours after hatching (hah); and the hatching percentage was estimated at 10 hah. The females ovulated between 5 and 6 hours $\left(28 \pm 1,0^{\circ} \mathrm{C}\right)$ and immediately the eggs were obtained by extrusion, as well as the semen. The absolute fecundity was $178331,6 \pm 28773,7$ oocytes/female and the relative $137,7 \pm 19,6 \mathrm{~g}$ oocytes/Kg of female. The diameter of the newly spawned oocytes was $687,2 \pm 42,8 \mu \mathrm{m}$. Fertility was $90,7 \pm 1,4 \%$ and hatching $85,3 \pm 1,5 \%$. The results allow to conclude that EPC at dosage of $6 \mathrm{mg} / \mathrm{Kg}$ of weight is a good inducer of the vizcaína reproduction, which high ovulation index and high rates of fertility and hatching.
\end{abstract}

Key words: Curimatidae; spawning; hatching; fecundity

\begin{abstract}
Resumo
Com o objetivo de reproduzir vizcaína Curimata mivartii, espécimenes de dois anos de idade $(\mathrm{n}=54)$, mantidos em tanques em terra $\left(0,1\right.$ peixes $\left./ \mathrm{m}^{2}\right)$ foram induzidos com extrato de hipófise de carpa (EPC) (Argent, USA) a uma taxa de 6 mg / $\mathrm{kg}$ de peso para as fêmeas em duas aplicações (10\% e 90\% com 12 horas entre aplicações), enquanto os machos foram induzidos com 4,8 mg / kg de EPC em dose única. Antes da indução hormonal para cada fêmea, uma biópsia ovariana foi realizada para verificar o seu estado de maturação gonadal. A amostra de oócitos foi tratada com solução de Serra (Chemi, Colômbia) e após três minutos, com auxílio de um estereoscópio de luz (Leica, EZ4 W, Alemanha) em objetivo 4x, foi determinada a posição da vesícula germinativa (central, migrando, periférico, sem nucleo). A análise da biópsia ovariana mostrou que as fêmeas estavam em maturação final. Os machos induzidos foram encontrados na fase de espermizaçã; que na pressão abdominal leve liberou fluido seminal. O índice de ovulação, fecundidade (absoluta e relativa), diâmetro do oócito e volume seminal foram estimados. A percentagem de fertilidade foi estimada em quatro horas pós-eclosão (hpe); enquanto a porcentagem de eclosão foi estimada em $10 \mathrm{hp}$. As fêmeas ovularam entre 5 e 6 horas $\left(28 \pm 1,0^{\circ} \mathrm{C}\right)$ e imediatamente os ovos foram obtidos por extrusão, assim como o sêmen. A fecundidade absoluta foi 178331,6 $6 \pm 28773,7$ oócitos

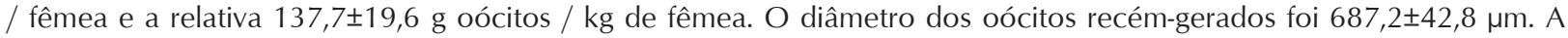
fertilidade foi de $90,7 \pm 1,4 \%$ e incubação de $85,3 \pm 1,5 \%$. Os resultados permitem concluir que a EPC na dosagem de $6 \mathrm{mg} /$ Kg de peso é um bom indutor de reprodução de vizcaína, com alto índice de ovulação e altas taxas de fertilidade e eclosão.
\end{abstract}

Palavras chave: Curimatidae; desova; incubação; fecundidade

\section{Introducción}

Vizcaína Curimata mivartii (Curimatidae), también conocida como cachaca, sardina o vizcaíno, crece hasta los 35 cm de longitud total; es endémica de Colombia y se registra en las ciénagas del bajo Magdalena (Mojica et al., 2012). Se le considera una especie que realiza migraciones locales cortas (Usma et al., 2009); aunque su patrón migratorio no ha sido plenamente establecido, se conoce que difiere de la generalidad de las especies migratorias de la cuenca magdalénica, al no hacer parte de la migración de principio de año conocida como subienda, sino que permanece en las ciénagas durante todo el periodo seco (Mojica et al., 2012).
De las 1494 especies de peces de agua dulce que existen en Colombia (DoNascimento et al., 2017), 81 se encuentran con algún grado de vulnerabilidad (Mojica et al., 2012) y la reproducción en cautiverio es una herramienta de conservación ex situ; pero solo algunas tienen tecnologías de reproducción en cautiverio como cachama negra Colossoma macropomum, bocachico Prochilodus magdalenae, dorada Brycon moorei, charúa Brycon sinuensis, yamú Brycon amazonicus, comelón Megaleporinus muyscorum, capaz Pimelodus grosskopfii, blanquillo Sorubim cuspicaudus y bagre rayado Pseudoplatystoma magdaleniatum. Sin embargo, especies como vizcaína Curimata mivartii, la cual se encuentra reportada como vulnerable según el libro 
rojo de peces dulce acuícolas de Colombia (Mojica y Usma, 2012), es de las menos estudiada, tanto así que su talla mínima de capturas aún no ha sido reglamentada. Ante la reducción en las capturas de bocachico y bagres (Mojica y Usma, 2012), el interés por otras especies nativas como recurso pesquero ha aumentado, como el caso de la vizcaína, lo que ha generado una disminución en el número de individuos de esta especie en las ciénagas y tributarios del río magdalena.

La reproducción es un proceso controlado por factores ambientales iniciales y finales tanto de tipo biótico (depredación, competición, proporción de sexo, densidad de población, entre otros) como abióticos (caudal, fotoperíodo, temperatura, $\mathrm{pH}$, oxígeno disuelto, conductividad eléctrica, entre otros) actúan en diferentes momentos del ciclo reproductivo (Avendaño-lbarra et al., 2004; Macedo-Soares et al., 2009; Atencio-García y Kerguelén-Durango, 2015). Los factores finales estimulan el apareamiento y desove y proporcionan condiciones favorables para la viabilidad de las crías (Baumgartner et al., 2008; Atencio-García y KerguelénDurango, 2015). Sin embargo el cautiverio ocasiona disfunción reproductiva y la más común en los peces nativos migratorios, caso vizcaína, es la inhibición de la ovulación y el desove por la ausencia de estos estímulos; por lo que es necesario inducciones hormonales para incrementar el volumen seminal en machos y la ovulación y desove en las hembras (Mylonas et al., 2010).

Ante la ausencia de estímulos ambientales finales en cautiverio para la ovulación y el desove en los peces, uno de los métodos para superar esta disfunción reproductiva es la hipofización (Atencio, 2001), siendo el extracto pituitario de carpa (EPC) uno de los inductores más utilizados (Yaron et al., 2009). Existen registros de reproducción de otros Characiformes con importancia comercial con EPC como bocachico (AtencioGarcía et al., 2013), cachama blanca (Díaz-Olarte et al., 2010; Castillo-Losada et al., 2016), cachama negra (Arias Acuña y Hernández Rangel, 2009), dorada (Atencio-García et al., 2017), charúa (Atencio-García et al., 2006; Montes y Salgado 2014), comelón (AtencioGarcía et al., 2001), picuda Salminus affinis (Weingartner y Zaniboni-Filho, 2010; Zanandrea et al., 2016), sábalo Prochilodus lineatus (Botta et al., 2010); lo que señala la efectividad del EPC al permitir la reproducción inducida de especies nativas migratorias que no desovan en cautiverio. Son escasos los reportes en la literatura evaluando la reproducción inducida en especies de la familia Curimatidae; por lo que el objetivo del presente estudio fue evaluar la reproducción inducida de vizcaína Curimata mivartii con EPC.

\section{Materiales y métodos}

\section{Localización}

El estudio se realizó en la Estación Piscícola San Silvestre S.A, entre septiembre - octubre de 2018, ubicada a $7^{\circ} 06^{\prime} 41.8$ "N y $73^{\circ} 51^{\prime} 23.0^{\prime \prime} \mathrm{O}$ (Barrancabermeja, Col), a una altitud de $92 \mathrm{msnm}$, con temperatura media anual fue de $28^{\circ} \mathrm{C}$ y humedad relativa del $75 \%$.

\section{Selección de reproductores $e$ inducción hormonal}

Ejemplares de dos años de edad ( $n=54)$ mantenidos en estanques en tierra a densidad de $0,1 \mathrm{pez} / \mathrm{m}^{2}$ fueron trasladados a piletas circulares $\left(3 \mathrm{~m}^{3}\right)$ con flujo constante de $(5 \mathrm{~L} / \mathrm{min})$, donde permanecieron durante 24 horas, con el propósito de habituarlos a las condiciones experimentales y reducir el estrés generado por la manipulación y cambio de ambiente.

Las hembras para inducción fueron seleccionadas, luego de verificar la presencia de ovocitos en maduración final, para lo cual se tomó una muestra de biopsia ovárica con una sonda naso-gástrica de Levin, calibre 6, de PVC grado médico (Medex, Colombia). La muestra de ovocitos fue tratada con solución Serra (Chemí, Colombia) y después de tres minutos, leídas con la ayuda de un estereoscopio de luz, (Leica, EZ4 W, Alemania) en objetivo de $4 x$, para determinar la posición de la vesícula germinal (céntrico, migrando, periférico, sin núcleos) y los ovocitos atrésicos (inviables) atendiendo los criterios sugeridos por Yaron et al., (2009). Los machos inducidos se encontraron en fase de espermiación; los cuales a ligera presión abdominal liberaron líquido seminal.

Las hembras fueron inducidas con EPC a una dosis de $6 \mathrm{mg} / \mathrm{Kg}$ en dos aplicaciones (10 y 90\% con 12 horas de diferencia); los machos fueron inducidos con una dosis única equivalente al $80 \%$ de la dosis de las hembras.

Los procedimientos empleados en la manipulación de los animales se realizaron atendiendo las normas y procedimientos para el uso de animales en laboratorio, señaladas por el Committe on Care and Use of Laboratory Animal Resources (National Research Council, USA 2010).

\section{Desempeño reproductivo}

El desempeño reproductivo se determinó mediante el índice de ovulación, fecundidad (absoluta y relativa) y porcentaje de fertilidad y eclosión. 
Índice de ovulación. Se consideró como el número de hembras ovuladas sobre el total de las hembras tratadas. La obtención de los productos sexuales se realizó mediante masaje en sentido cráneo caudal en la región abdominal.

Fecundidad absoluta. Para cada hembra se pesó el desove en una balanza digital (OHAUS, EC, USA). Luego se tomó una pequeña muestra entre 0,5 y 1,0 $\mathrm{g}$, se preservaron en formol $1 \%$ buferado para su conteo. La fecundidad absoluta (Fa), número de huevos desovados por hembra, se estimó con la fórmula: Fa $=\mathrm{n} \times \mathrm{G} / \mathrm{g}$; donde, $\mathrm{n}$ es el número de ovocitos en la submuestra, G el peso en gramo de los ovocitos desovados por hembra y g el peso de la submuestra.

Con los valores de Fa, se estimó la fecundidad relativa (Fr) dividiendo el peso total de ovocitos (g) sobre el peso de las hembras expresado en $\mathrm{Kg}$.

Para la determinación del diámetro de los ovocitos recién desovados, por lo menos 50 ovocitos del desove de cada hembra fueron medidos con la ayuda de un estereoscopio óptico (Leica, DM750, Suiza) y un analizador de imagen (Carl Zeiss, Axion visión 4.3, Alemania).

\section{Fertilidad y eclosión}

La incubación de los huevos se realizó en incubadoras cilindro-cónicas de $60 \mathrm{~L}$ de capacidad, con un flujo de 3,0 L/min. A las cuatro horas post-fertilización (hpf) se midió la tasa de fertilidad, tomando una muestra de 100 ovocitos (gastrulación final). Se consideraron como huevos viables aquellos que presentaron aspecto traslúcido y divisiones simétricas en la zona germinal; los no viables presentaron coloración blancuzca. El porcentaje de eclosión se evaluó entre las 10 y 12 hpf y se expresó como el porcentaje de embriones vivos y móviles (faringulación) en una muestra de aproximadamente 100 huevos.
Tabla 1. Calidad del agua en la incubación de huevos vizcaína Curimata mivartii.

\begin{tabular}{|l|c|}
\hline \multicolumn{1}{|c|}{ Parámetro } & Valor \\
\hline Temperatura $\left({ }^{\circ} \mathrm{C}\right)$ & $28 \pm 1,0$ \\
\hline $\mathrm{pH}$ & $7,7 \pm 0,3$ \\
\hline Oxígeno disuelto $(\mathrm{mg} / \mathrm{L})$ & $4,9 \pm 0,2$ \\
\hline Alcalinidad $\left(\mathrm{mg} / \mathrm{L} \mathrm{CaCO}_{3}\right)$ & $50 \pm 5$ \\
\hline Dureza $\left(\mathrm{mg} / \mathrm{L} \mathrm{CaCO}_{3}\right)$ & $25 \pm 5$ \\
\hline
\end{tabular}

El oxígeno disuelto $(\mathrm{mg} / \mathrm{L})$, temperatura $\left({ }^{\circ} \mathrm{C}\right), \mathrm{pH}$ fueron medidos con la ayuda de una sonda multiparamétrica (YSI Incorporated, Yellow Springs, OH, USA). Se determinó la dureza $\left(\mathrm{mg} / \mathrm{L} \mathrm{CaCO}_{3}\right)$ y la alcalinidad $\left(\mathrm{mg} / \mathrm{L} \mathrm{CaCO}_{3}\right)$ con un fotómetro digital (YSI, 9500, USA.)

\section{Análisis estadístico}

Se realizó estadística descriptiva y los resultados fueron expresados como promedio \pm desviación estándar.

\section{Resultados}

La tabla 1 registra los valores de la calidad del agua en la incubación de la vizcaína. El oxígeno disuelto se mantuvo por encima de $4,5 \mathrm{mg} / \mathrm{L}$ y la dureza y alcalinidad mostraron valores por encima de $25 \mathrm{mg} / \mathrm{L}$.

La tabla 2 registra las características de los reproductores inducidos hormonalmente con EPC. Las hembras inducidas presentaron peso promedio de $280,5 \pm 16,6$ g y longitud total (Lt) de 26,4 $\pm 0,9$ $\mathrm{cm} \quad(\mathrm{n}=18)$; mientras que los machos pesaron $171,2 \pm 13,8$ g y midieron $24,8 \pm 0,8 \mathrm{~cm}(\mathrm{n}=36)$. Aunque no se observó dimorfismo sexual; cuando los ejemplares están maduros las hembras se observan

Tabla 2. Características de los reproductores y de los productos sexuales de vizcaína Curimata mivartii, inducida con extracto pituitario de carpa.

\begin{tabular}{|l|c|c|}
\hline \multicolumn{1}{|c|}{ Características } & Hembras & Machos \\
\hline $\mathrm{n}$ & 18 & 36 \\
\hline $\mathrm{Lt}(\mathrm{cm})$ & $26,4 \pm 0,9$ & $24,8 \pm 0,8$ \\
\hline $\mathrm{Pt}(\mathrm{g})$ & $280,5 \pm 16,6$ & $171,2 \pm 13,8$ \\
\hline $\begin{array}{l}\text { Color } \\
\text { (ovocitos-semen) }\end{array}$ & Gris-marrón-marrón & Blanco \\
\hline Volumen seminal $(\mathrm{mL})$ & - & $0,1 \pm 0,1$ \\
\hline
\end{tabular}


con el abdomen abultado y la papila genital dilatada y enrojecida; mientras que en los machos el abdomen es anguloso (afilado).

La tabla 3 muestra los resultados de las biopsias ováricas de las hembras inducidas; en las cuales se observó que más del 60\% de los ovocitos presentaron vesícula germinal descentrada (migrando, periféricas y sin núcleo); lo cual corresponde a ovocitos en maduración final y por tanto las hembras inducidas se encontraron en estado de maduración final. Además se registró un bajo porcentaje de ovocitos atrésicos $(<8.0 \%)$.

Tabla 3. Biopsia ovárica de vizcaína Curimata mivartii inducida con extracto pituitario de carpa.

\begin{tabular}{|l|c|}
\hline $\begin{array}{c}\text { Posición de la vesícula } \\
\text { germinal (núcleo) }\end{array}$ & $\mathbf{( \% )}$ \\
\hline Céntricos (\%) & $7,9 \pm 4,3$ \\
\hline Migrando (\%) & $23,0 \pm 6,8$ \\
\hline Periféricos (\%) & $16,8 \pm 5,1$ \\
\hline Sin núcleo (\%) & $45,0 \pm 10,9$ \\
\hline Atrésicos (\%) & $7,3 \pm 2,5$ \\
\hline
\end{tabular}

La evaluación del desempeño reproductivo de la vizcaína inducida con EPC indica que la ovulación ocurrió entre las 5 a 6 horas post-inducción $\left(28,0 \pm 1^{\circ} \mathrm{C}\right)$, con un índice de ovulación del 90\%. La fecundidad relativa fue de $137,7 \pm 19,6$ g ovocitos/kg de hembra y la fecundidad absoluta estuvo alrededor de $178,331 \pm 28,773 \mathrm{~g}$ de ovocitos/hembra. La tasa de

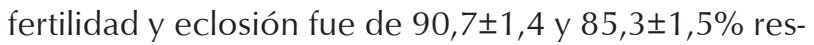
pectivamente (tabla 4).

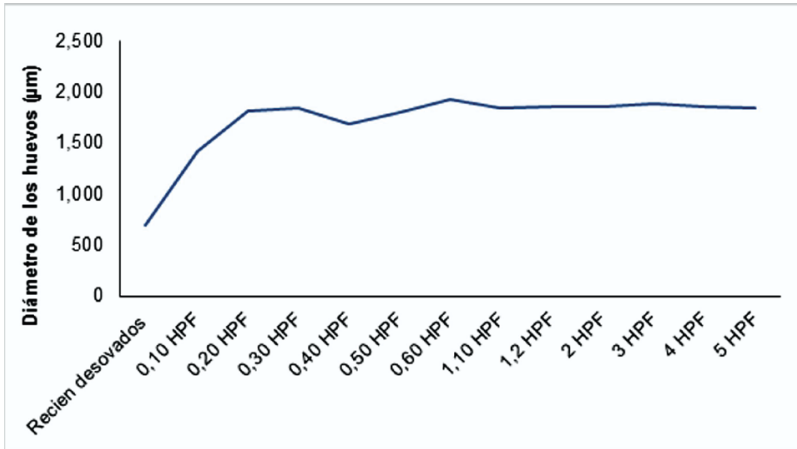

Figura 1. Curva de hidratación de los huevos de vizcaína en incubadoras de flujo ascendente. * Diámetro máximo y tiempo de hidratación.
Tabla 4. Desempeño reproductivo de vizcaína Curimata mivartii, inducida con extracto pituitario de carpa. ${ }^{*}$ hpi: horas post-inducción

\begin{tabular}{|l|c|}
\hline \multicolumn{1}{|c|}{ Parámetro } & Valor \\
\hline Dosis de aplicación (mg/kg) & 6 \\
\hline Tiempo de latencia (hpi) & $5-6$ \\
\hline Índice de ovulación (\%) & 90 \\
\hline Número de ovocitos/g & $4617 \pm 83$ \\
\hline $\begin{array}{l}\text { Fecundidad absoluta } \\
\text { (g de ovocitos/hembra) }\end{array}$ & $38,6 \pm 6,2$ \\
\hline $\begin{array}{l}\text { Fecundidad relativa (número } \\
\text { de ovocitos/hembra) }\end{array}$ & $178331,6 \pm 28773,7$ \\
\hline $\begin{array}{l}\text { Fecundidad relativa (g } \\
\text { ovocitos/kg de hembra) }\end{array}$ & $137,7 \pm 19,6$ \\
\hline Fertilidad (\%) & $90,7 \pm 1,4$ \\
\hline Eclosión (\%) & $85,3 \pm 1,5$ \\
\hline
\end{tabular}

Durante el proceso de incubación de los huevos se observó que los ovocitos recién desovados de vizcaína registraron diámetro de 687,2 $\pm 42,8 \mu \mathrm{m}$, con abundante vitelo (telolecítico), a las 0,20 hpf el diámetro aumentó 2,6 veces $(1808 \mu \mathrm{m})$, obteniéndose la máxima hidratación a las 0,60 hpf con un valor de $1923,9 \pm 112,8 \mu \mathrm{m}$ y un incrementó del diámetro 2.8 veces (figura 1 ).

\section{Discusión}

El agua del sistema de incubación se encontró dentro del rango adecuado para la incubación de peces neotropicales (Atencio-García et al., 2013; Mira et al., 2010; Valbuena-Villarreal et al., 2010); lo cual sugiere que el desempeño reproductivo (tasas de fertilidad y eclosión) no fueron afectados por la calidad del agua.

La vizcaína respondió adecuadamente al protocolo de inducción con EPC, registrando alto índice de ovulación $(90 \%)$, altas fecundidades y tasas de fertilización y eclosión (> 80\%). Uno de los aspectos cruciales en el éxito de la reproducción en cautiverio es la selección adecuada de los reproductores; lo cual es posible con una adecuada lectura de la biopsia ovárica. Las hembras de vizcaína inducidas se caracterizaron por presentar más del 60\% de los ovocitos en maduración final (sumatorias de ovocitos migrando, periféricos y sin núcleo). Algunos autores han sugerido que el éxito de una sustancia inductora depende en gran medida del porcentaje de ovocitos en maduración final que se determine en la biopsia ovárica; es así como en 
carpa común Cyprinus carpio, se sugirió inducir solo hembras con EPC cuando la presencia de ovocitos en maduración final (migrando, periféricos y sin núcleos), por los menos alcanzarán 66\% (Yaron et al., 2009); en bocachico Prochilodus magdalenae se ha establecido como mínimo el $70 \%$ de ovocitos en maduración final para obtener desoves exitosos (Montes-Petro, 2018). Lo resultados del presente estudio sugieren que para inducir la vizcaína con altas tasas de fertilidad y eclosión se deben seleccionar hembras con ovocitos con diámetro entre 650 y 750 um y por lo menos, con $60 \%$ de ovocitos en maduración final.

La alta fecundidad registrada por la vizcaína, indica que el $14 \%$ de su peso lo transforma en ovocitos, los cuales se caracterizan por presentar pequeño tamaño (menor de $700 \mu \mathrm{m}$ ), lo que corresponde a una especie con estrategia reproductiva r2 (Jiménez-Segura et al., 2009), tal particularidad ha sido observada en otras especies de carácidos como bocachico (Atencio et al., 2013), cachama negra (Arias-Acuña y HernándezRangel, 2009), cachama blanca (Chaves-Moreno et al., 2011), comelón (Atencio-García et al., 2001; SeguraGuevara et al., 2017).

En otras especies de la familia Curimatidae se han reportado diámetros de ovocitos maduros similares a los observados en el presente estudio. Andrade et al., (2010) en Curimatella lepidura registró diámetro de $711 \pm 44 \mu \mathrm{m}$. En carácidos de otras familias como Leporinus obtusidens (Anastomidae) el diámetro del ovocito maduro se estimó entre 515,2 a 963,0 um (Andrade et al., 2013); mientras que Peressin et al., (2012) trabajando con Cyphocharax nagelii e Steindachnerina insculpta reportaron diámetro de ovocitos maduros de $1025 \mu \mathrm{m}$ y $975 \mu \mathrm{m}$ respectivamente. En Megaleporinus muyscorum el diámetro del ovocito maduro se estimó en 929 $\pm 19 \mu \mathrm{m}$, con una fecundidad absoluta entre 31009 y 63900 ovocitos/hembra y $5171 \pm 865$ ovocitos por gramos (Atencio-García et al., 2001).

Atencio-García et al., (2013) reportaron para bocachico Prochilodus magdalenae, un pez reofílico con un alto valor comercial, una fecundidad absoluta de 192058 ovocitos/hembra y una fecundidad relativa de 160,5 \pm 51,0 g ovocitos/kg de hembra; mientras que Arias-Acuña y Hernández-Rangel (2009), para cachama negra Colossoma macropomum reportaron una fecundidad relativa entre 67,5 y 155,6 g ovocitos/kg de hembra. Estos resultados muestran que el diámetro de ovocito maduro y la fecundidad de la vizcaína, estimados en el presente estudio, se encuentra dentro del rango reportado para otros peces migratorios de los ríos suramericanos.
Durante el proceso de incubación de los huevos, la fase de hidratación es crítica, ya incrementa la flotabilidad de los huevos y la viabilidad del embrión; y luego de esta fase los huevos son más resistente a la manipulación y manejo. En la presente investigación se estableció que los huevos de vizcaína Curimata mivartii, registran su máximo diámetro de hidrata-

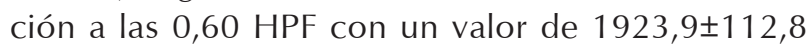
$\mu \mathrm{m}$. Valores muy similares a los obtenidos por diversos autores en especies reofílicas como en Prochilodus magdalenae (2230 $\mu \mathrm{m})$ (Arias-Gallo et al., 2010);

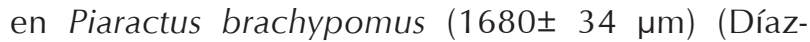
Olarte et al., 2010). En Siluriformes, también se han reportado sobre el diámetro máximo de hidratación de los huevos, como en capaz Pimelodus grosskopfii el diámetro de máxima hidratación es de $2700 \mu \mathrm{m}$ (Valbuena-Villarreal et al., 2012); en Pseudoplatystoma sp el diámetro máximo se estimó en 1930 440 um (Díaz-Olarte et al., 2010) y en Steindachneridion parahybae este diámetro se registró en $1900 \pm 60$ mm (Honji et al., 2012). La fuerte hidratación del huevo es una característica de los huevos de los peces reofílicos, por su condición de huevos semi-pelágicos (huevos de flotabilidad neutra) que requieren de un medio lótico para viabilizar el embrión. La vizcaína registra una fuerte hidratación en menos de una hora post-eclosión (0.6 hpe) y su diámetro total se incrementó 2,8 veces y el espacio perivitelínico se estimó en un $24 \%$ del tamaño total del huevo. Según Nakatani et al., (2001), los peces reofílicos del neotrópico se caracterizan por presentar una fuerte hidratación que le permite un amplio espacio perivitelínico (mayor al 20\%).

\section{Conclusiones}

Bajo condiciones de cautiverio la vizcaína Curimata mivartii puede ser inducida con EPC a razón de $6 \mathrm{mg} /$ $\mathrm{Kg}$ de peso, aplicado en dos dosis en las hembras (10 y $90 \%$ ) y una dosis única en los machos correspondiente al $80 \%$ de la dosis total de la hembra. Las hembras deben presenta huevos entre 650 y $750 \mu \mathrm{m}$ y el porcentaje de ovocitos en maduración final debe ser por lo menos del $60 \%$.

\section{Agradecimientos}

El Grupo de Investigación en Peces Nativos (GIPEN) adscrito a la Piscícola San Silvestre S.A, agradece a ISAGEN S.A. E.S.P por la financiación de la presente investigación en el marco del convenio 47-698. Y al Instituto de Investigación Piscícola de la Universidad de Córdoba (CINPIC). 


\section{Referencias}

Andrade D, Vidal JM, Godinho H, Tonini W, Burket D. Fecundidade da manjuba Curimatella lepidura (curimatidae) na represa de Três Marias, rio São Francisco, MG. Arq Bras Med Vet Zootec. 2010;62(6):1401-1408. http://dx.doi.org/10.1590/S010209352010000600016

Andrade E, Felizardo V, Murgas L, Drumond M, Zangerônimo M, Costa S. Reproductive dynamics of Leporinus obtusidens captured downstream from an important hydropower Station in Brazil. Arq Bras Med Vet Zootec. 2013;5(65): 1419-1426. http://dx.doi.org/10.1590/S0102-09352013000500021

Arias-Acuña J, Hernández-Rangel J. Efectos del extracto hipofisiario de carpa común y el análogo de la GnRH sobre la maduración final del oocito y el desove de la cachama negra (Colossoma macropomum). Revista Científica, FCV-LUZ. 2009;19(5):486-494. https://www.redalyc.org/articulo. oa?id=95911615008

Arias-Gallo $M$, Jiménez-Segura L, Dorado-Longas $M$ del P. Desarrollo larval de Prochilodus magdalenae (Steindachner, 1879) (Pisces: prochilodontidae), río Magdalena, Colombia Actual Biol. 2010;32(93):199-208. http://www.scielo.org.co/pdf/acbi/ v32n93/v32n93a7.pdf

Atencio-Garcia V, Arabia RF, Aristizabal J. 2006. Desarrollo embrionario y larvario de dorada (Brycon sinuensis). Comunic. Científ. CIVA. Pp. 604-618. On line: http://www.civa2006.org

Atencio-García V, Arguello L, González H. Reproducción inducida de la liseta Leporinus muyscorum (Steindachner, 1920) con extracto pituitario de carpa (EPC) Rev MVZ Córdoba. 2001;5(2):12. DOI: https://doi.org/10.21897/rmvz.529

Atencio-Garcia V, Dorado-Longas M, Montes-Petro C, Prieto-Guevara M, Espinosa-Araujo J. Cryopreservation of dorada (Brycon moorei) sperm with dimethyl sulfoxide. Rev. colomb. Biotecnol. 2017;19(2):87-94. http://dx.doi.org/10.15446/rev.colomb. biote.v19n2.59987

Atencio-Garcia V, Kerguelén-Durango E, Robert-Petro E. Desempeño reproductivo del bocachico (Prochilodus magdalenae) inducido dos veces en un mismo año. Rev MVZ Córdoba. 2013;18(1):3304-3310. www.scielo.org.co/pdf/mvz/v18n1/ v18n1a08.pdf

Atencio-García V, Kerguelén-Durango E. Environmental characterization of the reproductive season of migratory fish of the Sinú river (Córdoba, Colombia). Rev MVZ Córdoba. 2015;20(3):47664778. http://www.scielo.org.co/pdf/mvz/v20n3/v20n3a10.pdf

Atencio-García V. Producción de alevinos de especies nativas. Rev MVZ Córdoba. 2001;6(1):9-14.

Avendaño-lbarra R, Funes-Rodríguez R, Hinojosa-Medina A, González-Armas R, Aceves-Medina G. Seasonal abundance of fish larvae in a subtropical lagoon on in the west coast of the Baja California Peninsula. Estuar Coast Shelf Sci. 2004; 61(1):125135. https://doi.org/10.1016/j.ecss.2004.03.017

Baumgartner G, Nakatani K, Gomes LC, Bialetzki A, Sanches PV, Makrakis MC. Fish larvae from the upper Paraná River: do abiotic factors affect larval density Neotrop Ichthyol. 2008;6(4):551558. http://dx.doi.org/10.1590/S1679-62252008000400002
Botta P, Sciara A, Arranz S, Murgas L, Pereira G, Oberlender G. Estudio del desarrollo embrionario del sábalo (Prochilodus lineatus). Arch Med Vet. 2010;42: 109-114. http://dx.doi.org/10.4067/ S0301-732X2010000200014

Castillo-Losada E, Medina-Robles V, Cruz-Casallas P. Evaluación de la viabilidad de embriones de cachama blanca (Piaractus brachypomus) conservados a $-14{ }^{\circ} \mathrm{C}$ en diferentes estadios de desarrollo embrionario. Arch Med Vet. 2016; 48(1):69-77 http:// dx.doi.org/10.4067/S0301-732X2016000100009

Chaves-Moreno L, Chacón-Rodríguez L, Lozada-Morales J, MottaDelgado P, Murcia-Ordoñez B. Evaluación de la reproducción inducida de cachama blanca (Piaractus brachypomus) con acetato de buserelina. Vet Zootec. 2011;6(1):47-55. http://vip. ucaldas.edu.co/vetzootec/downloads/v6n1a05.pdf

Díaz-Olarte J, Marciales-Caro L, Cristancho VF, Cruz-Casallas PE. Comparación del desarrollo embrionario de Piaractus brachypomus (Serrasalmidae) y Pseudoplatystoma sp. (Pimelodidae). Int. J. Morphol. 2010;28(4):1193-1204. http://dx.doi.org/10.4067/ S0717-95022010000400033

DoNascimento C, Herrera-Collazos EE, Herrera-R GA, Ortega-Lara A, Villa-Navarro FA, Usma Oviedo JS, Maldonado-Ocampo JA. Checklist of the freshwater fishes of Colombia: A Darwin Core alternative to the updating problem. ZooKeys. 2017;708:25138. https://doi.org/10.3897/zookeys.708.13897

Honji R, Tolussi C, Mello P, Caneppele D, Moreira R. Embryonic development and larval stages of Steindachneridion parahybae (Siluriformes: Pimelodidae) - implications for the conservation and rearing of this endangered Neotropical species. Neotropical Ichthyology. 2012;10(2):313-327. http://dx.doi.org/10.1590/ S1679-62252012005000009

Jiménez-Segura L, Palacio J, López R. Características biológicas del blanquillo (Sorubim cuspicaudus) Littmann, Burr y Nass, 2000 y bagre rayado (Pseudoplatystoma magdaleniatum) Buitrago Suárez y Burr, 2007 (Siluriformes: Pimelodidae) relacionadas con su reproducción en la cuenca media del río Magdalena, Colombia. Actual Biol. 2009;31(90):53-66. http://www.scielo. org.co/pdf/acbi/v31n90/v31n90a5.pdf

Macedo-Soares L, Birolo A, Freire A. Spatial and temporal distribution of fish eggs and larvae in a subtropical coastal lagoon, Santa Catarina State, Brazil. Neotropical Ichthyology. 2009;7(2):231-240. http://dx.doi.org/10.1590/S167962252009000200015

Mira T, Medina-Roble, Cruz-Casallas P. Evaluación de tres protocolos hormonales para la inducción de la espermiación en yaque (Leiarius marmoratus). Rev. MVZ Córdoba. 2010;15(2):20702077. http://www.scielo.org.co/scielo.php?script=sci_abstract \&pid=S0122-02682010000200005

Mojica JI, Lasso C, Sánchez P, Usma JS, Álvarez R. (eds.). 2012. Libro rojo de peces dulceacuícolas de Colombia. Serie Libros Rojos de Especies Amenazadas de Colombia. Instituto de Ciencias Naturales - Universidad Nacional de Colombia y Ministerio del Medio Ambiente. Bogotá, Colombia. p. 237-239.

Mojica JI, Usma JS. 2012. Curimata mivartii. En: Mojica JI, Lasso C, Sánchez P, Usma JS, Álvarez R. (eds.). Libro rojo de peces dulceacuícolas de Colombia. Serie Libros Rojos de Especies Amenazadas de Colombia. Instituto de Ciencias Naturales - 
Universidad Nacional de Colombia y Ministerio del Medio Ambiente. Bogotá, Colombia. Pp. 107-108.

Montes C, Salgado S. 2014. Efecto de la concentración de glucosa sobre la movilidad espermática de la dorada (charúa) Brycon sinuensis (Trabajo de pregrado). Departamento Ciencias Acuícolas, Facultad de Medicina Veterinaria y Zootecnia, Universidad de Córdoba, Montería.

Montes-Petro C. 2018. Evaluación del desempeño reproductivo del bocachico (Prochilodus magdalenae) (Steindachner 1878), en cautiverio mediante predictores tempranos de calidad espermática y ovocitaria. Trabajo de grado, Facultad de Ciencias Básicas. Maestría en Biotecnología, Universidad de Córdoba, Montería, Colombia.

Mylonas C, Fostier A, Zanuy S. Broodstock management and hormonal manipulations of fish reproduction. Gen Comp Endocrinol. 2010; 165:516-534. DOI: 10.1016/j.ygcen.2009.03.007

Nakatani K, Agostino AA, Baumgartner G, Bialetzky A, Sanches PV, Makrakis MC, Pavanelli CS. 2001. Ovos e larvas de peixes de agua doce: desenvolvimento e manual de identifação. Maringa: Eduem. p.377

Peressin A, Gonçalves C, Braga De Souza F. Reproductive strategies of two Curimatidae species in a Mogi Guaçu impoundment, upper Paraná River basin, São Paulo, Brazil. Neotropical Ichthyology. 2012;10(4):847-854. http://dx.doi.org/10.1590/S167962252012000400018

Segura-Guevara F, López-Corrales H, De La Hoz C, Olaya-Nieto W. Reproductive biology of Liseta Leporinus muyscorum Steindachner, 1901 in the Sinú River, Colombia Rev MVZ Córdoba. 2017;22(1):5728-5737. http://dx.doi.org/10.21897/rmvz.932
Usma JS, Valderrama M, Escobar MD, Ajiaco-Martínez RE, Villa-Navarro F, Castro F, et al. 2009. Peces dulceacuícolas migratorios en Colombia. Pp. 103 - 131. En: Amaya, J. D. y L. G. Naranjo (Eds.). Plan Nacional de las Especies Migratorias: Diagnóstico e identificación de acciones para la conservación y el manejo sostenible de las especies migratorias de la biodiversidad en Colombia. MAVDT - WWF. Bogotá D. C. Colombia. p.214

Valbuena VR, Zapata B, Ruales CD, Cruz-Casallas PE. Desarrollo embrionario del capaz (Pimelodus grosskopfii) (Steindachner, 1879). Int J Morphol. 2012; 30(1):150-156. http://dx.doi. org/10.4067/S0717-95022012000100027

Valbuena-Villarreal R, Zapata-Berruecos B, Cruz-Casallas P. Reproducción inducida de capaz (Pimelodus grosskopfii) con extracto de hipófisis de carpa: reporte preliminar. Orinoquia. 2010;14(2):133-139. http://www.scielo.org.co/pdf/rori/ v14n2/v14n2a03.pdf

Weingartner M, Zaniboni-Filho E. 2010. Biologia e cultivo do Dourado. In B. Baldisserotto, \& L. C. Gomes, (Eds), Espécies Nativas para Piscicultura no Brasil. Santa Maria, RS: Editora UFSM. Pp. 245-281.

Yaron Z, Bogomolnaya A, Drori S, Biton I, Aizen J, Kulikovsky Z, Levavi-Sivan B. Spawning Induction in the Carp: Past Experience and Future Prospects - A Review. Rev The Israeli Journal of Aquaculture - Bamidgeh. 2009;61(1):5-26. https://www. researchgate.net/publication/263280621_Spawning_Induction_in_the_Carp_Past_Experience_and_Future_Prospects__A_Review.

Zanandrea-volpato A, Weingartner M, Zaniboni-Filho E. Induced reproduction of dourado (Salminus brasiliensis): fertilization with sperm cryopreserved in $A C P ®-104$. Acta Sci Anim Sci. 2016;(38):1. http://dx.doi.org/10.4025/actascianimsci. v38i1.28838
César Montes: https://orcid.org/0000-0002-6930-3494 Víctor Atencio: https://orcid.org/0000-0002-2533-1995 Ana Estrada: https://orcid.org/0000-0003-3585-3719 Jonny Yepes: https://orcid.org/0000-0001-6276-5488 